\title{
Efeitos da laserterapia de baixa intensidade na cicatrização de úlceras nos pés em pessoas com diabetes mellitus
}

\section{Effects of low-level laser therapy on the healing of foot ulcers in people with diabetes mellitus}

\section{Efectos de la terapia con láser de bajo nivel sobre la curación de las úlceras del pie en personas con diabetes mellitus}

\begin{abstract}
Maria Girlane Sousa Albuquerque Brandão', Maria Aline Moreira Ximenes², Aline de Oliveira Ramalho3, Vivian Saraiva Veras ${ }^{1}$, Lívia Moreira Barros ${ }^{1}$, Thiago Moura de Araújo ${ }^{1 *}$
\end{abstract}

ORCID IDs

Brandão MGSA (DD https://orcid.org/0000-0002-9925-4750

Ximenes MAM (D) https://orcid.org/0000-0002-1674-3357

Ramalho AO (D) https://orcid.org/0000-0001-6065-5488

Veras VS (D) https://orcid.org/0000-0003-3267-3712

Barros LM (iD https://orcid.org/0000-0002-0174-2255

Araújo TM (D) https://orcid.org/0000-0002-8410-0337

\section{COMO CITAR}

Brandão MGSA; Ximenes MAM; Ramalho AO; Veras VS; Barros LM; Araújo TM (2020) Efeitos da laserterapia de baixa intensidade na cicatrização de úlceras nos pés em pessoas com diabetes mellitus. ESTIMA, Braz. J. Enterostomal Ther., 18: e0320. https://doi. org/10.30886/estima.v18.844_PT

\section{RESUMO}

Objetivo: Identificar os efeitos da laserterapia de baixa intensidade na cicatrização do pé diabético. Método: Revisão sistemática nas bases de dados PubMed, LILACS, SciELO, CINAHL, Cochrane, Web of Science e Scopus, em que foram identificados 73 artigos, dos quais seis foram incluídos na amostra final, após verificação dos critérios de elegibilidade. Resultados: Os artigos apontaram como efeitos da laserterapia a efetividade na progressão do processo de reparo tecidual do pé diabético, alívio da dor, ação antiinflamatória, aumento da perfusão tecidual da lesão e melhora da resposta vascular e do sistema nervoso. Conclusão: 0 laser é uma terapia adjuvante que pode acelerar o processo de cicatrização da lesão, diminuir a dor, melhorar a neovascularização e, assim, minimizar o risco de complicações, como amputação do membro inferior e melhora da qualidade de vida de pessoas com diabetes e integridade da pele prejudicada.

DESCRITORES: Terapia a laser; Cicatrização de feridas; Pé diabético; Estomaterapia.

\begin{abstract}
Objective: Identify the effects of low-level laser therapy on the healing of diabetic foot. Method: Systematic review of the PubMed, LILACS, SciELO, CINAHL, Cochrane, Web of Science and Scopus databases, in which 92 articles were identified and six were included in the final sample after the eligibility criteria. Results: The articles pointed out as effects of laser therapy the effectiveness in the progression of the tissue repair process of the diabetic foot, pain relief, anti-inflammatory action, increased tissue perfusion of the lesion and improvement of the vascular response and the nervous system. Conclusion: Laser is an adjuvant therapy that can
\end{abstract}

1.Universidade da Integração Internacional da Lusofonia Afro-Brasileira - Departamento de Enfermagem - Redenção (CE), Brasil.

2.Universidade Federal do Ceará - Departamento de Enfermagem - Fortaleza (CE), Brasil.

3.Hospital Sírio-Libanês - Serviço de Estomaterapia - São Paulo (SP), Brasil.

*Autor correspondente: thiagomoura@unilab.edu.br

Recebido: Jan. 18, 2020 | Aceito: Mar. 09, 2020 
accelerate the wound healing process, relieve pain, improve neovascularization, and thus minimize the risk of complications, such as lower limb amputation and improvement of quality of life for people with diabetes and impaired skin integrity.

DESCRIPTORS: Laser therapy; Wound healing; Diabetic foot; Stomatherapy.

\section{RESUMEN}

Objetivo: Identificar los efectos de la terapia con láser de bajo nivel en la curación del pie diabético. Método: Revisión sistemática en las bases de datos PubMed, LILACS, SciELO, CINAHL, Cochrane, Web of Science y Scopus, en las que se identificaron 73 artículos, de los cuales seis se incluyeron en la muestra final, después de verificar los criterios de elegibilidad. Resultados: Los artículos señalaron como efectos de la terapia con láser la efectividad en la progresión del proceso de reparación de tejidos del pie diabético, alivio del dolor, acción antiinflamatoria, aumento de la perfusión tisular de la lesión y mejora de la respuesta vascular y el sistema nervioso. Conclusión: El láser es una terapia adyuvante que puede acelerar el proceso de curación de la lesión, disminuir el dolor, mejorar la neovascularización y, por lo tanto, minimizar el riesgo de complicaciones, como la amputación de miembros inferiores y la mejora de la calidad de vida de las personas con diabetes e integridad de la piel deteriorada.

DESCRIPTORES: Terapia con láser; Curación de heridas; Pie diabético; Estomaterapia.

\section{INTRODUÇÃO}

O diabetes mellitus (DM) é uma das doenças crônicas mais prevalentes no mundo e um dos maiores desafios de saúde pública do século XXI ${ }^{1}$. Dentre as complicações mais frequentes do DM, estão as úlceras de pé diabético².

O pé diabético é causado principalmente por neuropatia periférica e doença arterial, que levam à ulceração do pé. Essa ulceração pode progredir com infecção e/ou destruição de tecidos moles, que sucede em déficits de deambulação e impacto considerável na qualidade de vida, além de recursos gastos com hospitalização prolongada, reabilitação, assistência domiciliar e custos diretos e indiretos ao paciente, suas famílias e a sociedade, devido à perda de produtividade ${ }^{3,4}$.

Aproximadamente 15\% das pessoas com DM sofrerão com úlceras nos pés em sua vida útil, em que os tratamentos convencionais, como limpeza e curativo diário com soro fisiológico 0,9\%, ácidos graxos essenciais, hidrogel e solução de betadina demonstraram pouco sucesso no progresso do reparo tecidual, com maior índice de complicações ${ }^{5-7}$.

Uma coorte realizada com 1055 pessoas com DM em 19 centros especializados no Brasil evidenciou que 24,2\% apresentavam úlcera prévia nos pés e $13,7 \%$ amputação anterior, e, destes, $5,3 \%$ possuíam mais de uma amputação.

Assim, nos últimos anos, terapias adjuvantes foram testadas para estimular o processo de cura de feridas em pessoas com $\mathrm{DM}^{5}$. Entre as novas terapias, a laserterapia de baixa intensidade (LBI) é apontada como um método não invasivo, indolor, de baixo custo e com eficácia no tratamento de feridas, por atuar nos eventos fisiológicos e bioquímicos do processo de cicatrização ${ }^{9-11}$.
Estudos disponíveis sobre aplicação de laserterapia para tratamento de úlceras nos pés em pessoas com DM ainda são escassos. Embora existam relatos favoráveis à sua aplicação, novas investigações devem ser realizadas a fim de obter os efeitos do laser no tratamento dessas lesões, bem como identificar o número de aplicações, dose e comprimento de onda necessários para a reparação tecidual, o que reforça a relevância do estudo.

Justifica-se a pesquisa pela ocorrência elevada de DM na população nos últimos anos e o aumento de casos de amputações não traumáticas nos membros inferiores, o que destaca a necessidade de haver não apenas o tratamento da doença de base, mas também a prevenção de lesões e o acompanhamento do pé diabético com outras terapias adjuvantes que possam favorecer o progresso cicatricial.

Este estudo tem como objetivo identificar os efeitos da laserterapia de baixa intensidade na cicatrização do pé diabético.

\section{MÉTODO}

Trata-se de revisão sistemática da literatura, que buscou identificar, selecionar e analisar a produção científica sobre efeitos da laserterapia na cicatrização do pé diabético. As revisões sistemáticas utilizam métodos sistematizados e explícitos com finalidade de selecionar e avaliar criticamente pesquisas relevantes ${ }^{12}$.

O levantamento bibliográfico foi realizado entre janeiro e junho de 2019. Para coletar os dados, utilizou-se a estratégia PICO, cujo acrônimo significa: $P-$ Population, aponta a população 
em foco, $I$ - Intervention, determina o tipo de intervenção, $C$ - Comparison, identifica qual grupo-controle será testado com a intervenção, e, $O$ - Outcome, os desfechos que serão avaliados. Essa estratégia ajuda a formular a questão de pesquisa e possibilita a identificação de palavras-chave que auxiliam na localização de estudos primários relevantes nas bases de dados ${ }^{13}$.

Diante do exposto, a questão norteadora deste estudo, pautada na estratégia PICO, ilustrada na Tabela 1, é: "quais os efeitos da laserterapia de baixa intensidade na cicatrização do pé diabético?”.

Dada a peculiar temática e o número pequeno de artigos científicos na literatura sobre o assunto, a busca foi atemporal, nas bases de dados: National Library of Medicine (PubMed), Literatura Latino-Americana e do Caribe em Ciências da Saúde (LILACS), Scientific Electronic Library Online (SciELO), Cumulative Index to Nursing and Allied Health Literature (CINAHL), Cochrane Library, Web of Science e Scopus, disponibilizadas diretamente em seus sites ou pelo Portal Capes, realizada por dois pesquisadores independentes.

Tabela 1. Esquema ilustrativo do processo de elaboração da questão norteadora com base no acrônimo PICO.

\begin{tabular}{cc}
\hline População & Pessoas com pé diabético \\
\hline Intervenção & Laserterapia \\
Comparação & Terapia convencional \\
Desfecho & Cicatrização \\
\hline
\end{tabular}

A estratégia de busca adotada foi a utilização de descritores e operador booleano "AND". Os descritores também foram selecionados pautando-se na estratégia PICO e identificados por meio dos Descritores em Ciências da Saúde (DECS): Terapia com luz de baixa intensidade and Cicatrização de feridas and Pé diabético; e por meio do Medical Subject Headings (MeSH): Low-level light therapy and Wound Healing and Diabetic Foot.

Como critérios de inclusão foram considerados estudos experimentais, disponibilizados eletronicamente nos idiomas inglês, português e espanhol, que realizaram o tratamento do pé diabético com LBI. Foram excluídos experimentos realizados com lesões de outras etiologias ou com animais, artigos de revisão com seres humanos ou outras espécies animais e estudos in vitro.

Foram identificados 92 artigos nas sete bases de dados consultadas. Após o levantamento das publicações, foram excluídos os artigos duplicados ( $\mathrm{n}=38)$. Em seguida, os títulos foram analisados e os resumos foram lidos e categorizados segundo os critérios de inclusão e exclusão preestabelecidos, perfazendo uma amostragem final de seis artigos, após a análise de dois pesquisadores, conforme a Fig. 1.

Para certificar a qualidade metodológica dos artigos incluídos, utilizou-se a recomendação PRISMA, que consiste em um checklist com 27 itens de análises sistemáticas e metanálises ${ }^{14}$. Todas as produções científicas utilizadas foram devidamente citadas e referenciadas, em respeito aos direitos autorais de pesquisa.

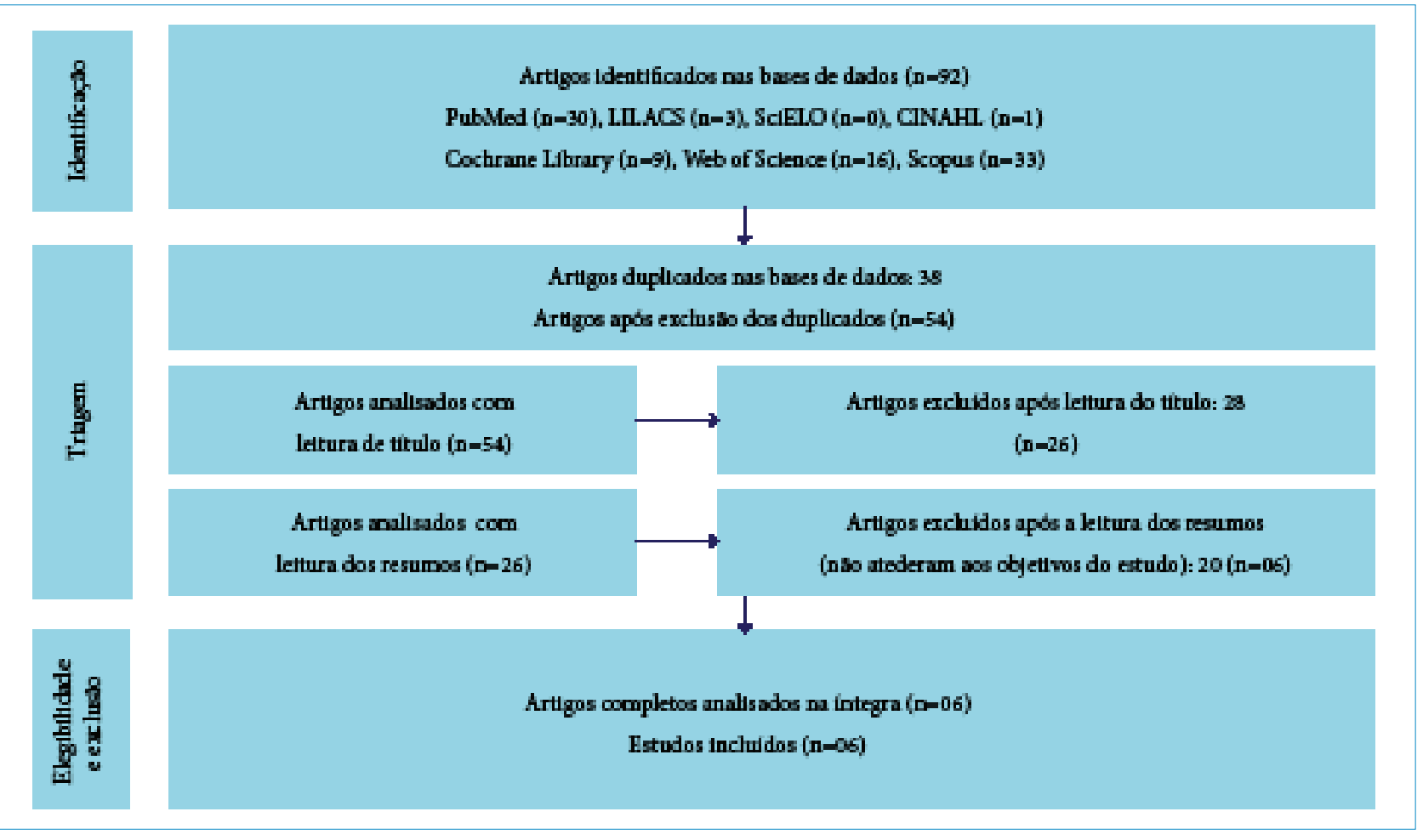

Figura 1. Fluxograma ilustrativo do processo de seleção dos estudos segundo a recomendação PRISMA. 


\section{RESULTADOS}

\section{Perfil das publicações}

Os seis artigos incluídos na revisão estão apresentados na Tabela 2, os quais tiveram como objetivo analisar os efeitos da laserterapia no processo de cicatrização do pé diabético.

Com relação ao ano de publicação, tem-se um artigo datado de 2018, dois de 2017, um de 2016, um de 2015 e um de 2011. Quanto ao idioma, todas as publicações estão em idioma inglês.

Os estudos selecionados foram desenvolvidos nos seguintes países: Brasil $(n=3)$, Índia $(n=1)$, Itália $(n=1)$ e Irã $(n=1)$. Quanto à categoria do autor principal das publicações, duas pertencem a fisioterapeutas, duas a médicos, uma a enfermeira e um a biomédico.

\section{Protocolo de tratamento}

O número de participantes dos estudos variou de 16 a 56 pacientes. Em cinco estudos os pacientes foram alocados aleatoriamente em grupos diferentes: grupo-controle e grupo-intervenção.

$\mathrm{Na}$ pesquisa de Santos et al. $(2018)^{6}$, as lesões no grupo-controle foram tratadas com solução fisiológica 0,9\% e hidrogel. No estudo de Carvalho et al. (2016) ${ }^{15}$, os participantes foram distribuídos aleatoriamente em quatro grupos: controle (limpeza e curativo simples), terapia a laser de baixa intensidade, ácidos graxos essenciais (AGE) de Calendula officinalis e laser associado ao AGE. No estudo de Feitosa et al. $(2015)^{16}$, os participantes do grupo-controle realizaram curativo simples com solução fisiológica 0,9\%; já Mathur et al. (2017) ${ }^{17}$ utilizaram solução de betadina. Os demais estudos não informaram o tratamento utilizado no grupo-controle.

O comprimento de onda em nanômetros (nm) utilizado para aplicação do laser variou de 632,8 a $830 \mathrm{~nm}$. As doses em joules $(\mathrm{J})$ foram de três a $20 \mathrm{~J} / \mathrm{cm}^{2}$. No estudo de Salvi et al. $(2018)^{18}$, a quantidade de aplicações do laser na ferida e o tempo de acompanhamento não foram especificados. Em quatro estudos foram realizadas 12 sessões de laserterapia para tratamento do pé diabético ${ }^{6,7,15,16}$.

O estudo de Santos et al. $(2018)^{6}$ realizou as sessões de laser de 48/48 h em um período de quatro semanas. No estudo de Mathur et al. (2017) ${ }^{17}$, as feridas foram submetidas a exposição à luz laser diariamente por 15 dias. Em Carvalho et al. (2016) ${ }^{15}$ e Feitosa et al. $(2015)^{16}, 12$ sessões foram realizadas, compreendendo três atendimentos semanais, em dias alternados. $\mathrm{Na}$ pesquisa realizada por Kaviani et al. $(2011)^{7}$, os pacientes receberam radiação com laser sobre as feridas seis vezes por semana, durante pelo menos duas semanas consecutivas e depois a cada dois dias até completar a cura.

\section{Avaliação da ferida}

A avaliação do processo cicatricial do pé diabético tratado com LBI foi realizada por meio de métodos diversos: aplicação da escala de úlcera de pressão para cicatrização (PUSH, do inglês pressure ulcer scale for healing) ${ }^{6}$, mensuração da evolução da ferida por meio de fita adesiva com divisão em centímentros ${ }^{15} \mathrm{e}$ avaliação de registros fotográficos ${ }^{7,16,17}$.

\section{Benefícios}

Os artigos apontaram como principal resultado da laserterapia no pé diabético a eficácia na progressão do processo de reparo tecidual em menor período de tempo, além de outros efeitos como alívio da dor, ação anti-inflamatória, maior perfusão tecidual da lesão e melhora na resposta dos sistemas vascular e nervoso.

\section{DISCUSSÃO}

A partir da análise dos resultados, observou-se o consenso em relação ao uso da LBI para favorecer a progressão do reparo tecidual de úlceras por pé diabético. Ademais, favorece o alívio da dor, maior perfusão tecidual da lesão, estímulo da neovascularização e proliferação celular quando comparado com grupos-controle que utilizaram ácidos graxos essenciais de Calendula officinalis, solução fisiológica 0,9\%, hidrogel e solução de betadina.

Vale salientar que não houve registros de eventos adversos associados ao tratamento em questão e todos os estudos demonstraram resultados positivos no processo de reparação tecidual com o uso de LBI em comparação com grupos-controle.

$\mathrm{O}$ estudo realizado no Irã constatou que, na quarta semana de tratamento com LBI, o tamanho das feridas nos pés de pessoas com diabetes diminuiu significativamente e, após 20 semanas, houve cicatrização completa ${ }^{7}$.

Pesquisa realizada na Índia evidenciou que, em duas semanas de terapêutica com LBI, as úlceras nos pés de $75 \%$ dos pacientes do grupo de tratamento tiveram redução de 30-50\% da área lesionada e maior quantidade de tecido de 
Tabela 2. Descrição dos estudos incluídos na revisão sistemática.

\begin{tabular}{|c|c|c|c|c|c|c|c|}
\hline Título/Base de dado & $\begin{array}{c}\text { Autores/ } \\
\text { Ano }\end{array}$ & Objetivo & $\begin{array}{l}\text { Método/ } \\
\text { Amostra }\end{array}$ & $\begin{array}{l}\text { Comprimento } \\
\text { de onda } \\
(\mathrm{nm})\end{array}$ & $\begin{array}{c}\text { Dose } \\
\left(\mathrm{j} / \mathrm{cm}^{2}\right)\end{array}$ & $\begin{array}{l}\text { Número de } \\
\text { aplicações }\end{array}$ & $\begin{array}{l}\text { Resumo dos } \\
\text { resultados obtidos }\end{array}$ \\
\hline $\begin{array}{l}\text { Effects of low-power } \\
\text { light therapy on the } \\
\text { tissue repair process } \\
\text { of chronic wounds in } \\
\text { diabetic feet. } \\
\text { (PubMed) }\end{array}$ & $\begin{array}{l}\text { Santos } \\
\text { et al. } \\
2018^{6}\end{array}$ & $\begin{array}{c}\text { Analisar a } \\
\text { eficácia do uso } \\
\text { terapêutico do } \\
\text { laser de baixa } \\
\text { potência no } \\
\text { reparo tecidual } \\
\text { do pé diabético. }\end{array}$ & $\begin{array}{l}\text { Ensaio clínico } \\
\text { randomizado } \\
18 \text { pacientes }\end{array}$ & 660 & 6 & $\begin{array}{c}12 \\
\text { aplicações } \\
\text { em } 4 \\
\text { semanas }\end{array}$ & $\begin{array}{l}\text { Alívio da dor e eficácia } \\
\text { na progressão do } \\
\text { processo de reparo } \\
\text { tecidual em curto } \\
\text { período }(p<0,013) \text {. }\end{array}$ \\
\hline
\end{tabular}

Low-level laser therapy as an adjunct

to conventional

therapy in the treatment of diabetic foot ulcers. (PubMed)
Mathur do laser de baixa

et al.

$2017^{17}$

tratamento do pé potência no diabético.
Ensaio clínico

randomizado

30 pacientes
15

aplicações

em 2

semanas
Eficácia na progressão

do processo de reparo

tecidual em curto

período ( $p=0,009)$.

\section{(}

Effect of low-level

light therapy on

diabetic foot ulcers:

a near-infrared

spectroscopy study.

(PubMed)
Avaliar a eficácia

do laser de baixa

Salvi potência na

et al. modificação dos

$2017^{18}$ sistemas vascular

e nervoso em

feridas nos pés.
Ensaio clínico

randomizado

56 pacientes
Maior perfusão tecidual da lesão após a aplicação, melhora na resposta dos sistemas vascular e nervoso, eficácia na progressão do processo de reparo tecidual em curto

período $(p=0,017)$.

\section{Low-level laser therapy and}

calendula officinalis

in repairing diabetic foot ulcers.

(PubMed)
Avaliar os efeitos

Carvalho et al. $2016^{15}$ do laser de baixa potência no reparo de feridas em pé diabético.
Ensaio clínico

randomizado

32 pacientes
830

20

especificado

\section{Effects of the Low-}

Level Laser Therapy

(LLLT) in the process

of healing diabetic

foot ulcers

(PubMed)

$\begin{array}{cc} & \text { Avaliar os efeitos } \\ \text { Feitosa } & \text { do laser de baixa } \\ \text { et al. } & \text { intensidade no } \\ 2015^{16} & \text { reparo tecidual } \\ & \text { do pé diabético. }\end{array}$

Ensaio clínico

randomizado

16 pacientes
Alívio da dor $(p<0,001)$, ação anti-inflamatória, redução da área total das úlceras ( $p=0,0032$ ), estímulo da neovascularização e proliferação celular.

\section{A randomized clinical}

trial on the effect of low-level laser therapy on chronic diabetic foot wound healing: a preliminary report.

(Cochrane)
Avaliar a eficácia

Kaviani da terapia laser

et al. de baixa potência

$2011^{7}$ no tratamento do pé diabético.
Ensaio clínico

randomizado

23 pacientes
12

632,8

4 aplicações

em 4

semanas
Reparação tecidual no pé diabético $(p<0,005)$, biomodulação e efeito analgésico ( $p<0,001)$. 
granulação ${ }^{17}$.Pesquisadores brasileiros identificaram diferença estatística significante no índice de reparo tecidual de lesões em pessoas com diabetes após sessões de tratamento com $\mathrm{LBI}^{6}$.

Em outros estudos houve redução estatisticamente significante da dor pós-laser, mediante a avaliação da dor por meio da escala analógica visual, que pontua de 0 a 10 os níveis de dor, em que houve redução de média 9 na escala para 5 após o uso do laser ${ }^{15,16}$.

Percebe-se, portanto, que, além de contribuir na redução do tempo do tratamento, o laser é uma terapêutica coadjuvante que pode favorecer alívio no estado álgico e no desconforto local. A diminuição da dor pode impactar na qualidade de vida e possibilitar que o paciente esteja mais ativo em suas atividades de vida diária.

A aplicação do laser também promoveu aumento de neovascularização e proliferação de fibroblastos, com diminuição na quantidade do infiltrado inflamatório nas lesões, o que contribui para a modulação do reparo tecidual com cicatrização mais rápida e organizada ${ }^{15,18}$. Por meio desse processo, o laser pode recrutar citocinas e fatores de crescimento importantes para estimular a cicatrização da ferida ${ }^{19}$.

Não houve unanimidade nos estudos com relação ao comprimento de onda utilizado durante as sessões de LBI na cicatrização do pé diabético. Contudo cinco estudos apresentaram comprimento de onda entre 632,8 e $685 \mathrm{~nm}^{6,7,15,16,17}$.

Estudiosos sul-africanos evidenciaram que feridas em pessoas com diabetes irradiadas com laser em comprimento de onda próximo a 632,8 nm apresentaram maior grau de crescimento e migração de células bem como luminescência de adenosina trifosfato (ATP) ${ }^{20}$. Estudo de revisão que integrou pesquisas sobre a eficácia da LBI em úlceras resultantes de DM identificou que a maioria dos estudos utilizou comprimento de onda de $632,8 \mathrm{~nm}$ durante as sessões de tratamento com laser ${ }^{21}$.

Também não houve unanimidade com relação à dose em joules, porém a maioria dos estudos aplicou dosimetria de 3 a $6 \mathrm{~J} / \mathrm{cm}^{2}$ na área de comprometimento tecidual ${ }^{6,15-17}$. Revisão sistemática europeia que reuniu 20 estudos sobre os parâmetros de aplicação do laser em feridas diabéticas revelou que o uso de $4 \mathrm{~J} / \mathrm{cm}^{2}$ foi mais efetivo na aceleração da taxa de fechamento da ferida, redução da ação inflamatória, aumento da deposição de colágeno e maior proliferação de miofibroblastos ${ }^{22}$. Esse dado se mostra relevante para desenvolver e aperfeiçoar uma técnica eficiente de LBI para cicatrização do pé diabético, pois é essencial determinar os mecanismos exatos de ação e interação do laser com o organismo, com seleção adequada dos parâmetros de dosimetria e comprimento de onda.

Estudos na América Latina e Ásia realizaram 12 sessões de LBI para tratamento do pé diabético ${ }^{6,7,15,16,}$ valor suficiente para acelerar o reparo tecidual da ferida e promover os resultados esperados ${ }^{23}$. Contudo é válido inferir a importância da educação em saúde com o cliente durante a terapia, principalmente sobre a manutenção do controle glicêmico, cuidado com os pés e uso de calçados apropriados, além do estilo de vida saudável, para coadjuvar com o sucesso da terapêutica por fonte de luz.

Assim, a LBI pode ser vista como um método terapêutico efetivo na cicatrização de feridas, quando determinados fatores, como dose, potência, tempo e intervalo entre as sessões, são adequadamente observados. Ademais, a terapia a laser tem a vantagem de ser facilmente administrada. Os benefícios do uso do laser auxiliam na promoção da qualidade de vida do cliente e podem minimizar possíveis complicações, como o risco de amputação de membros inferiores 24 .

Os profissionais da saúde, em especial enfermeiros, desenvolvem importante papel nos cuidados de assistência às pessoas com feridas, portanto é fundamental que busquem habilitação para aplicar novas terapêuticas/tecnologias e, assim, conquistar melhorias na qualidade de vida de pessoas com diabetes e integridade da pele prejudicada.

\section{CONCLUSÃO}

Os achados do estudo apontam como efeitos da laserterapia o progresso na cicatrização do pé diabético, a melhora no estado álgico, o aumento na perfusão tecidual da lesão, que favorecem maior aporte sanguíneo e nutrição da área lesionada, além do estímulo da neovascularização e da proliferação celular, quando comparada com o uso de ácidos graxos essenciais de Calendula officinalis, solução fisiológica 0,9\%, hidrogel e solução de betadina.

Não houve unanimidade com relação à dose e ao comprimento de onda mais indicados para o tratamento do pé diabético. Contudo os indícios apontados nas produções científicas encontradas indicam que a utilização de laser com comprimento de onda próximo a $632,8 \mathrm{~nm}$ e dose de $4 \mathrm{~J} / \mathrm{cm}^{2}$ são os mais eficientes na terapêutica em estudo. 
Salienta-se a limitação na quantidade de pesquisas publicadas sobre pé diabético e LBI, sendo, portanto, de extrema relevância a realização de novos estudos que apresentem resultados cada vez mais promissores sobre o uso do laser no tratamento de feridas e que esclareçam os estágios de cura, a determinação científica dos parâmetros influentes de dose e o comprimento de onda, com rigorosos critérios de estudo para validar os dados de eficácia e segurança demonstrados nesta revisão.

\section{CONTRIBUIÇÃO DOS AUTORES}

Conceitualização, Brandão MGSA, Ximenes MAM, Ramalho AO, Veras VS, Barros LM e Araújo TM; Metodologia, Brandão MGSA, Ximenes MAM e Barros LM; Investigação, Brandão MGSA, Ximenes MAM; Redação - Primeira versão, Brandão MGSA, Ximenes MAM, Ramalho AO, Veras VS, Barros LM e Araújo TM; Redação - Revisão \& Edição, Brandão MGSA, Ximenes MAM e Araújo TM; Supervisão, Barros LM e Araújo TM.

\section{REFERÊNCIAS}

1. Zimmet P, Alberti KG, Magliano DJ, Bennett PH. Diabetes mellitus statistics on prevalence and mortality: facts and fallacies. Nat Rev Endocrinol. 2016;12(10):616-22. https:// doi.org/10.1038/nrendo.2016.105

2. Padilha AP, Rosa LM, Schoeller SD, Junkes C, Mendez CB, Martins MMFPS. Manual de cuidados às pessoas com diabetes e pé diabético: construção por scoping study. Texto Contexto Enferm. 2017;26(4):1-11. https://doi. org/10.1590/0104-07072017002190017

3. Morey-Vargas OL, Smith SA. BE SMART: Strategies for foot care and prevention of foot complications in patients with diabetes. Prosthet Orthot Int. 2015;39(1):48-60. https://doi. org/10.1177/0309364614535622

4. Salomé GM, Bueno JC, Ferreira LM. Aplicativo multimídia em plataforma móvel para tratamento de feridas utilizando fitoterápicos e plantas medicinais. Rev Enferm UFPE On Line. 2017;11(11 Supl):4579-88

5. Priyadarshini MJL, Kishore BEP, Thariq IA. Effect of low level laser therapy on diabetic foot ulcers: a randomized control trial. Int Surg J. 2018;5(3):1008-15. https://doi. org/10.18203/2349-2902.isj20180821

6. Santos JAF, Campelo MBD, Oliveira RA, Nicolau RA, Rezende VEA, Arisawa EÂL. Effects of low-power light therapy on the tissue repair process of chronic wounds in diabetic feet. Photomed Laser Surg. 2018;36(6):298-304. https://doi. org/10.1089/pho.2018.4455

7. Kaviani A, Djavid GE, Ataie-Fashtami L, Fateh M, Ghodsi M, Salami M, et al. A randomized clinical trial on the effect of lowlevel laser therapy on chronic diabetic foot wound healing: a preliminary report. Photomed Laser Surg. 2011;29(2):10914. https://doi.org/10.1089/pho.2009.2680

8. Parisi MCR, Moura Neto A, Menezes FH, Gomes MB, Teixeira RM, Oliveira JEP, et al. Baseline characteristics and risk factors for ulcer, amputation and severe neuropathy in diabetic foot at risk: the BRAZUPA study. Diabetol Metab Syndr. 2016;8(25):1-8. https://doi.org/10.1186/s13098-016-0126-8
9. Palagi S, Severo IM, Menegon DB, Lucena AF. Laser therapy in pressure ulcers: evaluation by the pressure ulcer scale for healing and nursing outcomes classification. Rev Esc Enferm USP. 2015;49(5):820-6. https://doi.org/10.1590/S0080623420150000500017

10. Kazemikhoo N, Vaghardoost R, Dahmardehei M, Mokmeli S, Momeni M, Nilforoushzadeh MA, et al. Evaluation of the effects of low level laser therapy on the healing process after skin graft surgery in burned patients (a randomized clinical trial). J Lasers Med Sci. 2018;9(2):139-43. https://doi. org/10.15171/jlms.2018.26

11. Gupta A, Keshri GK, Yadav A, Gola S, Chauhan S, Salhan AK, Singh SB. Superpulsed (Ga-As, 904 nm) low-level laser therapy (LLLT) attenuates inflammatory response and enhances healing of burn wounds. J Bio. 2015;8(6):489-501. https://doi.org/10.1002/jbio.201400058

12. Lima ACD, Cunha DA, Albuquerque RC, Costa RNA, Silva HJ. Alterações sensoriais em respiradores orais: revisão sistemática baseada no método prisma. Rev Paul Pediatr. 2018;37(1):97-103. https://doi.org/19840462/;2019;37;1;00012

13. Stillwell S, Fineout-Overholt E, Bernadette Melnyk B, Williamson K. Evidence-based practice, step by step: asking the clinical question: a key step in evidence-based practice. Am J Nurs. 2010;110(3):58-61. https://doi.org/10.1097/01. NAJ.0000368959.11129.79

14. Moher D, Liberati A, Tetzlaff J, Altman DG, The PRISMA Group. Preferred reporting items for systematic reviews and metaanalyses: the PRISMA Statement. PLoS Med. 2009;6(7):1-6. https://doi.org/10.1371/journal.pmed.1000097

15. Carvalho AFM, Feitosa MCP, Coelho NPMF, Rebêlo VCN, Castro JG, Sousa PRG, et al. Low-level laser therapy and Calendula officinalis in repairing diabetic foot ulcers. Rev Esc Enferm USP. 2016;50(4):628-34. https://doi.org/10.1590/ S0080-623420160000500013 
16. Feitosa MCP, Carvalho AFM, Feitosa VC, Coelho IM, Oliveira RA, Arisawa EÂL. Effects of the low-level laser therapy (LLLT) in the process of healing diabetic foot ulcers. Acta Cir Bras. 2015;30(12):852-7. https://doi.org/10.1590/S0102865020150120000010

17. Mathur RK, Sahu K, Saraf S, Patheja P, Khan F, Gupta PK. Low-level laser therapy as an adjunct to conventional therapy in the treatment of diabetic foot ulcers. Lasers Med Sci. 2017;32(2):275-82. https://doi.org/10.1007/s10103016-2109-2

18. 18. Salvi M, Rimini D, Molinari F, Bestente G, Bruno A. Effect of low-level light therapy on diabetic foot ulcers: a near-infrared spectroscopy study. J Biomed Opt. 2017;22(3):38001. https://doi.org/10.1117/1.JBO-22.3.038001

19. Tchanque-Fossuo CN, Ho D, Dahle SE, Koo E, Li C-S, Isseroff RR, Jagdeo J. A systematic review of low-level light therapy for treatment of diabetic foot ulcer. Wound Repair Regen. 2016;24(2):418-26. https://doi.org/10.1111/wrr.12399
20. Houreld N, Abrahamse H. Low-intensity laser irradiation stimulates wound healing in diabetic wounded fibroblast cells (WS1). Diabetes Technol Ther. 2010;12(12):971-8. https://doi.org/10.1089/dia.2010.0039

21. Sousa RG, Batista KNM. Laser therapy in wound healing associated with diabetes mellitus - Review. An Bras Dermatol. 2016;91(4):48993. https://doi.org/10.1590/abd1806-4841.20163778

22. Beckmann KH, Meyer-Hamme G, Schröder S. Low level laser therapy for the treatment of diabetic foot ulcers: a critical survey. Evid-Based Complementary Altern Med. 2014;2014(626127):19. https://doi.org/10.1155/2014/626127

23. Abdelbagi AM, Yousif MM, Yousif SM, Baloo MM. Effects of laser therapy on chronic skin ulcers healing interventions for Sudanese patients. Int J Res Med Sci. 2015;3(12):3720-5. https://doi.org/10.18203/2320-6012.ijrms20151429

24. Bavaresco T, Osmarin VM, Pires AUB, Moraes VM, Lucena AF. Low-power laser therapy in wound healing. J Nurs UFPE on line. 2019;13(1):216-26. https://doi.org/10.5205/1981-8963v13i1a235938p216-226-2019 